\title{
An exponential similarity measure for collaborative filtering
}

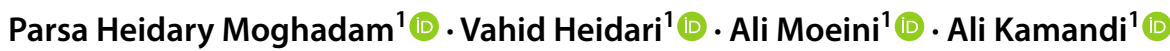

(c) Springer Nature Switzerland AG 2019

\begin{abstract}
In this paper, we propose two exponential similarity measures for collaborative filtering in recommender systems. The proposed similarity measures are used to estimate the distance between two users or items. Furthermore, an algorithm is proposed to use the distance obtained from similarity measures introduced in this paper to evaluate empty rating elements in the rating matrix and compare the results of these similarity measures with our exponential similarity measures. Therefore, we deduce that the algorithm using the exponential similarity measures works better than the algorithm using Manhattan, Euclidean, Jaccard, Cosine or Pearson similarity measures when taking K-Most-Similar-Users to evaluate the similarity in order to find ratings for the user that we want to predict ratings for.
\end{abstract}

Keywords Cosine similarity - Pearson similarity - Manhattan similarity - Euclidean similarity - Jaccard similarity . Collaborative filtering $\cdot$ Recommender systems $\cdot$ Data mining $\cdot$ Movielens

\section{Introduction}

Recently, big data is becoming an interest among researchers in computer science and other related fields. Recommender systems are groups of techniques that allow filtering through big data in order to give suggestion to users when needed.

Recommendation approaches for recommender systems are divided into three main aspects of content base, collaborative filtering, and hybrid filtering. Collaborative filtering recommendation systems use techniques which use similarity between users and items to find ratings based on those similarities and use them in order to suitably recommend to users.

One approach in collaborative filtering is called memory-based collaborative filtering which results in finding similar users or items to the current user or item and use their ratings to predict a rating for the current user or item based on similarity measures.
This paper will focus on memory-based collaborative filtering and introduces two novel exponential similarity measures in order to compute ratings for a user from ratings of most similar users to that particular user.

In Sect. 3, we will present our exponential similarity measures and explain why do they function better than popular similarity measures such as Manhattan, Euclidean, Jaccard, Cosine and Pearson [1]. The difference between our two exponential similarity measures is compared as well. Furthermore, an algorithm will be introduced to use any similarity measure in order to estimate ratings for empty elements of the rating matrix. The algorithm is solely for creating a platform to compare similarity measures with each other. Section 4 will bring RMSE (root mean squared error) results of our exponential similarity measures compared to Manhattan, Euclidean, Jaccard, Cosine and Pearson similarity measures by using the algorithm mentioned.

Parsa Heidary Moghadam, parsa.moghadam@ut.ac.ir; Vahid Heidari, vahid.heidari@ut.ac.ir; Ali Moeini, moeini@ut.ac.ir; Ali Kamandi, kamandi@ut.ac.ir| ${ }^{1}$ School of Engineering Sciences, College of Engineering, University of Tehran, Tehran, Iran. 


\section{Related work}

Collaborative filtering recommender system methods provide recommendations to any user, based on what other similar users have rated. Two kinds of collaborative filtering methods are model-based collaborative filtering and memory-based collaborative filtering [2-4]. Model-based collaborative filtering methods develop predictions based off of models that are created by data mining and machine learning techniques, whereas memory-based collaborative filtering approaches handle predictions by finding most similar users (or items) to the current user (or item) that we want to predict ratings for, and does this by means of using those similar user ratings [5]. We can use memory-based collaborative filtering methods using whereas Euclidean distance is the ordinary straightline distance between two points. Another similarity measure is Jaccard similarity measure which is basically an intersection over union between two vectors $[1,9]$.

\section{Proposed exponential similarity measures}

In this section, we propose a memory-based collaborative filtering algorithm which can accept any similarity formula to compute user-to-user similarity. This algorithm uses any similarity measure to predict a rating for any particular user. For RMSE results, we have used this algorithm to compare similarity measures together.

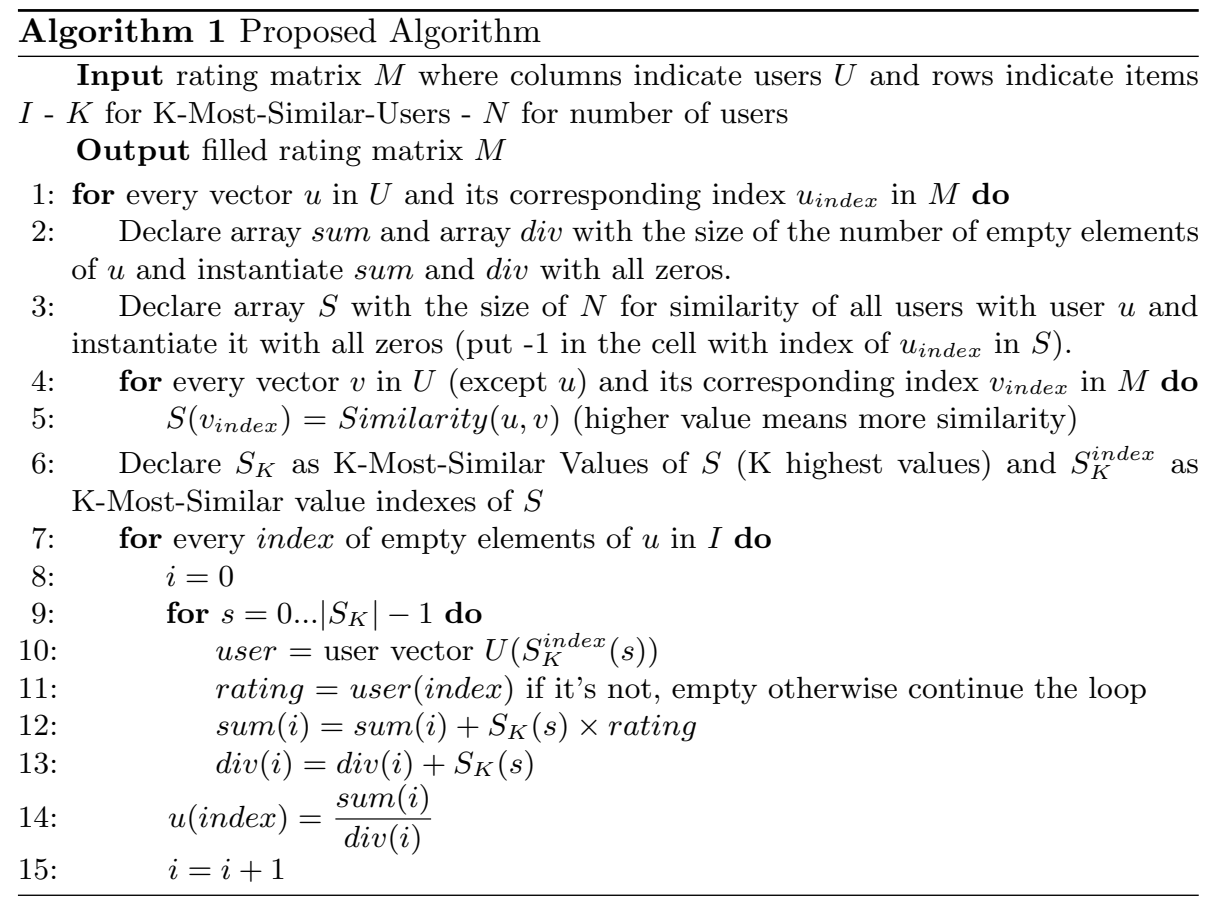

both user similarity [6] or item similarity $[7,8]$. In this article, we use user similarity to predict ratings. There are many measures used to find the similarity between users and items. One of these methods is Cosine similarity measure which measures the angle between two vectors in the space. A normalized version of this similarity measure was introduced as Pearson. There are other similarity measures such as Manhattan and Euclidean. Manhattan distance is the sum of the horizontal and vertical distances between points on a grid,
In Algorithm 1, we have used Manhattan, Euclidean, Jaccard, Cosine, Pearson and our novel similarity measures as a replacements for Simialrity function to calculate RMSE and compare the results of each similarity measure.

The exponential similarity measure that we introduce is an exponential distance measure which handles vectors that have empty elements. The formula is: 
Fig. 1 Exponential similarity measure

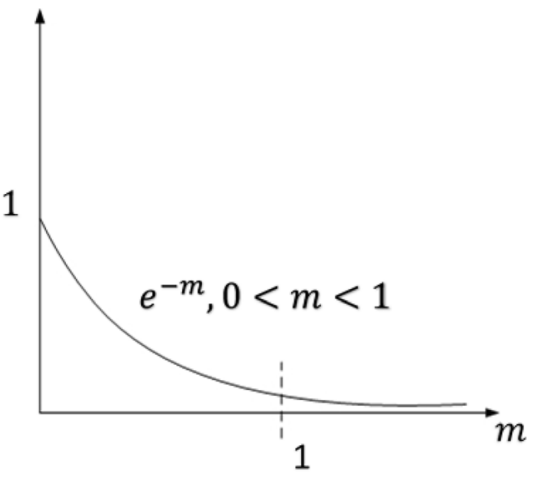

(a) Exponential similarity function

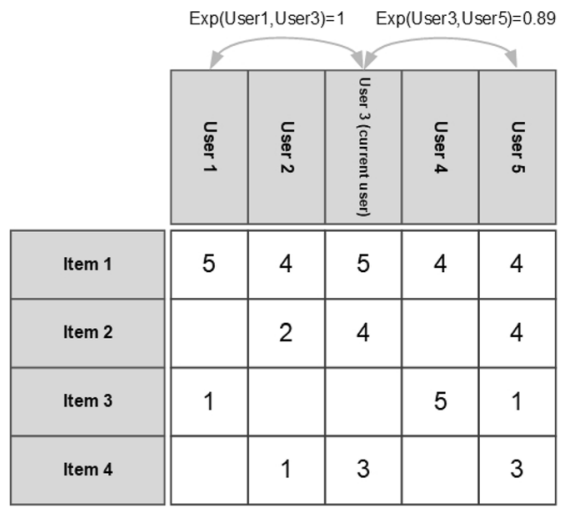

(b) Problem with exponential similarity measure
$\operatorname{Exp}\left(u_{i}, u_{j}\right)=e^{\frac{\sqrt{\sum_{\text {item=1 }}^{|/|}\left(u_{i}(\text { item })-u_{j}(\text { item })\right)^{2}}}{\phi \sqrt{f}}}$

where $0 \leq \operatorname{Exp}\left(u_{i}, u_{j}\right) \leq 1$ and $\phi$ is equal to the subtraction of the maximum available rating value and the minimum available rating value that a user can give and || $\mid$ is the number of items. Moreover, $f$ is the number of items rated by both users that we are finding similarity between (number of filled elements in both vectors).

As it is shown in Fig. 1a, the similarities are calculated in a smooth manner (smooth function) which can cause fair coefficients as a similarity measure.

The distance between two vectors in the rating matrix is measured in a way that only intersecting filled elements (elements filled by both user vectors for which we are finding similarity) of the two vectors (users) are getting compared, which can help with finding fair similarities and the exponential nature of this similarity measure causes the similarity coefficient to obey a smooth function. But the problem with this similarity measure is the unbalanced nature of it, which is the outcome of different numbers of intersecting filled elements between vectors which can result in unfair similarity coefficients. For instance, as it can be inferred from Fig. $1 \mathrm{~b}$ user 3 and user 1 are taken as the most similar users with the similarity coefficient of one. But since these two users have only one filled element in common, we cannot assume that they are completely similar since user 3 has other filled elements that are not intersecting with filled elements of user 1. On the other hand, in reality, user 3 and user 5 are the most similar users, since they have many intersecting filled elements which are very close in value. So the empty element of user 3 will be 0.89 if we are taking 1-MostSimilar-User which is not an optimal rating.

For solving this problem, we introduce another novel similarity measure that is a normalized version of the exponential similarity measure which pays attention to the number of intersecting filled elements between two vectors. So, now by using Formula 2 in Fig. 1b, user 3 and user 1 are not completely similar since user 3 has not rated the same items as user 1 (they have low number of intersecting elements). The formula of the novel normalized exponential similarity measure is as below:

$\operatorname{Exp-Normalized~}\left(u_{i}, u_{j}\right)=\frac{e^{\frac{\sqrt{\sum_{\text {item }=1}^{|| \mid}\left(u_{i}(\text { item })-u_{j}(\text { item })\right)^{2}}}{\phi \sqrt{f}}}}{\frac{\left|U_{i}\right|}{f}}$

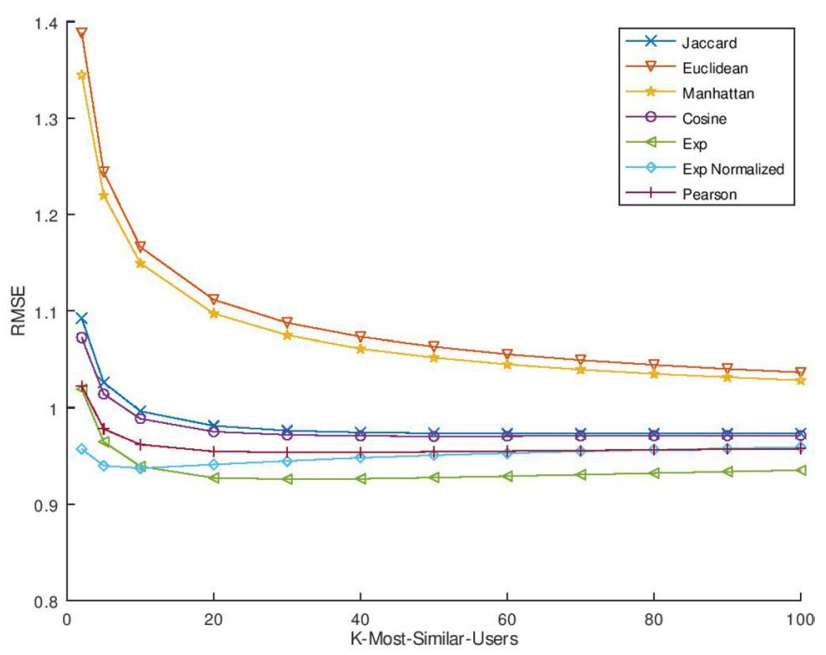

Fig. 2 RMSE results for similarity measures 
Table 1 RMSE results for different similarity measures

\begin{tabular}{lcccccc}
\hline RMSE for memory-based collaborative filtering \\
\hline Similarity method & $K=2$ & $K=5$ & $K=10$ & $K=20$ & $K=30$ & $K=100$ \\
\hline Manhattan & 1.3446 & 1.2199 & 1.1493 & 1.0972 & 1.0748 & 1.0284 \\
Euclidean & 1.3880 & 1.2440 & 1.1661 & 1.1115 & 1.0876 & 1.0365 \\
Jaccard & 1.0923 & 1.0262 & 0.9963 & 0.9811 & 0.9761 & 0.9732 \\
Cosine & 1.0726 & 1.0141 & 0.9885 & 0.9751 & 0.9718 & 0.9713 \\
Pearson & 1.0224 & 0.9779 & 0.9617 & 0.9546 & 0.9536 & 0.9574 \\
Exponential & 1.0192 & 0.9646 & 0.9390 & 0.9272 & 0.9258 & 0.9351 \\
Normalized exponential & 0.9572 & 0.9398 & 0.9371 & 0.9410 & 0.9447 & 0.9590 \\
\hline
\end{tabular}

where $\phi$ is equal to the subtraction of the maximum available rating value and the minimum available rating value that a user can give and || $\mid$ is the number of items. Moreover, $f$ is the number of elements rated by both users that we are finding similarity between (intersecting filled elements). $\left|U_{i}\right|$ is the number of filled elements in the current user vector that we are finding ratings for.

\section{Results}

In this section, we will bring the results of Algorithm 1 using all the similarity measures mentioned in Sect. 3 and calculating the RMSE to compare our exponential similarity measures with popular Manhattan, Euclidean, Jaccard, Cosine and Pearson similarity measures. For the results, we have used MovieLens dataset. The Movielens datasets consist of 5 stars ratings and tagging from https ://movielens.org. Each user has rated at least 20 movies and is presented with only one feature. This dataset is of 1, 10, 20 and 26 million records. Our results are estimated by a million record ratings MovieLens dataset. It consists of 6041 users and 3884 movies. For calculating RMSE, we have used $80 \%$ of the data for the train set and $20 \%$ for the test set.

In Table 1, $\mathrm{K}$ is the number of most similar users that we use, to predict ratings for the current user. We give this variable as an input to the algorithm proposed in the previous section. As it can be inferred from Table 1 and Fig. 2, the results can show improvement in our novel exponential similarity measures over popular similarity measures such as Manhattan, Euclidean, Jaccard, Cosine and Pearson. Furthermore, as $\mathrm{K}$ starts with a low number, we can see that our novel normalized exponential similarity measure works a lot better than other similarity measures, and as $\mathrm{K}$ gets larger our exponential similarity measure works better than other methods. We can infer that by using a small K for K-Most-Similar-Users which can also be found by many clustering techniques, we can bring down the time complexity of rating prediction with an acceptable precision.

\section{Compliance with ethical standards}

Conflict of interest The authors declare that they have no conflict of interest.

\section{References}

1. Su X, Khoshgoftaar TM (2009) A survey of collaborative filtering techniques. Adv Artif Intell 2009. http://dx.doi. org/10.1155/2009/421425

2. Tan Z, He L (2017) An efficient similarity measure for user-based collaborative filtering recommender systems inspired by the physical resonance principle. IEEE Access 5:27211-27228

3. Al Hassanieh L, Jaoudeh CA, Abdo JB, Demerjian J (2018) Similarity measures for collaborative filtering recommender systems. In: 2018 IEEE Middle East and North Africa communications conference (MENACOMM). IEEE, pp 1-5

4. Yi M, Xiao N, Tang R, Luo L, Yin X (2019) An efficient similarity measure for collaborative filtering. Procedia Comput Sci 147:416-421

5. Breese JS, Heckerman D, Kadie C (1998) Empirical analysis of predictive algorithms for collaborative filtering. In: Proceedings of the fourteenth conference on uncertainty in artificial intelligence. Morgan Kaufmann Publishers Inc., pp 43-52

6. Jin R, Chai JY, Si L (2004) An automatic weighting scheme for collaborative filtering. In: Proceedings of the 27 th annual international ACM SIGIR conference on research and development in information retrieval. ACM, pp 337-344

7. Deshpande $M$, Karypis $G$ (2004) Item-based top-n recommendation algorithms. ACM Trans Inf Syst (TOIS) 22(1):143-177

8. Linden G, Smith B, York J (2003) Amazon. com recommendations: item-to-item collaborative filtering. IEEE Internet Comput $1: 76-80$

9. Bagchi S (2015) Performance and quality assessment of similarity measures in collaborative filtering using mahout. Procedia Comput Sci 50:229-234

Publisher's Note Springer Nature remains neutral with regard to jurisdictional claims in published maps and institutional affiliations. 